Aim of the study: Abnormalities in signaling as well as altered gene expression have been identified in numerous diseases, including cancer. The biological functions of signal transducer and activator of transcription 3 (STAT3) are very broad. It is thought that STAT3 can also contribute to oncogenesis. RNA interference (RNAi) is one of the most efficient tools for silencing gene expression within cells. The main goal of the study was to verify the effectiveness of STAT3 gene silencing and its influence on cell proliferation and activation of apoptosis in bladder cancer cells.

Material and methods: The study was conducted on cellular material, which was the stable human bladder cancer cell line T24. The synthesis of shRNA (short hairpin RNA) interfering with the STAT3 gene was based on PSUPER. neo expression vector. The gene expression at the mRNA level was determined by the real-time PCR method. The influence of STAT3 gene silencing on apoptosis induced in cells with modulated STAT3 expression was evaluated using parallel quantification of mono- and oligonucleosomal DNA degradation of genomic DNA.

Results: In transfected T24 cells, the STAT3 mRNA expression decreased to the level of $68.3 \%$ compared to the scrambled (SCR) control. Silencing the STAT3 gene induced changes in the phenotype of T24 cells. Statistically significant differences in cell proliferation $(p=0.0318)$ and apoptosis induction $(p=0.0376)$ were observed.

Conclusions: Application of the designed shRNA for the STAT3 gene contributed to a decrease of expression of the examined gene. It also decreased the proliferation and increased the susceptibility to apoptosis in T24 bladder cancer cells.

Key words: RNA interference, shRNA, signal transducers and activator of transcription, apoptosis.

\section{The efficiency of silencing expression of the gene coding STAT3 transcriptional factor and susceptibility of bladder cancer cells to apoptosis}

\author{
Ilona Bednarek, Daniel Sypniewski, Natalia Gawlik, Anna Galilejczyk, \\ Karol Goraus
}

Department of Biotechnology and Genetic Engineering; The School of Pharmacy and Division of Laboratory Medicine; Medical University of Silesia in Katowice, Poland

\section{Introduction}

Despite the systematic development of diagnostics and treatment of many neoplastic diseases, there is still an unresolved problem with controlling and blocking the excessive proliferation of cancer cells. This process is connected with increase or constitutive expression of individual genes in the cancer cells. The most often overexpressed genes are those which are involved in survival, intensive proliferation and cells' defensive system, for example genes which control the process of apoptosis.

One of the most essential factors in the process of carcinogenesis is STAT proteins (signal transducer and activator of transcription). In normal human cells, STAT proteins are inactive, cytoplasmic transcriptional factors, which can be activated by extracellular signal molecules, for example growth factors, cytokines, hormones and peptides [1-3].

STAT3 signaling pathways play an important role in physiological processes, such as organogenesis, embryogenesis, innate and acquired immune response, regulation of differentiation of the cells, growth and apoptosis [4-10]. Among STAT proteins, STAT3 has a pleiotropic nature and can be activated by many cytokines, including interleukin 6 (IL-6), IL-10 and interferon I (IFN I). Depending on cell type, STAT3 can lead to apoptosis or can promote cell survival. The key role of STAT3 in the process of carcinogenesis is connected with the fact that overexpression of STAT proteins can indirectly lead to the malignant transformation and expansion of cancer cells, through intensive proliferation and apoptosis inhibition [11]. Limiting or completely silencing the expression of the genes coding transcriptional factors seems to be a promising strategy in anticancer therapy.

The silencing of gene expression can be done on both transcriptional and post transcriptional levels. RNA interference (RNAi) is an innovative technique which creates an opportunity for using nucleic acids as anticancer drugs, which can post transcriptionally suppress the expression of selected target genes [12]. Using RNAi, activation of the molecular degradation of selected transcripts is possible. The process is based on intercellular amplification of short, antisense, ribonucleic sequences [13].

In the present work, the RNAi phenomenon has been used for post transcriptional silencing of expression of the gene coding the STAT3 protein. We have also examined the susceptibility of bladder cancer cells, transfected with gene-specific siRNAs (small interfering RNAs), to apoptosis in the presence of topoisomerase-etoposide inhibitor. 


\section{Material and methods}

\section{Designing shRNA and cloning it into vector}

The target interfering sequence for shRNA, silencing the selected gene (STAT3), was designed using programs from the EMBOSS software package [14]. From a group of designed sequences we chose one which fulfilled the following requirements: the chosen sequence was localized about 50 to 100 nucleotides above the initiation of the translation region; we avoided regions located near the start codon (AUG) and noncoding sequences (5' or 3' UTR, untranslated region), which are usually rich in sequences binding regulatory proteins. As a result, the RISC complex has difficult access to target mRNA and efficiency of the whole process could be low; the analyzed sequence should contain 30-50\% of $\mathrm{G} / \mathrm{C}$ pairs; sequences rich in guanine were avoided, as they create quaternary structures. Furthermore, it was checked whether chosen sequences were complementary as regards other genes. In this case, we confirmed that the designed sequence would silence only our chosen STAT3 gene [15].

The designed sequence for STAT3 shRNA was cloned into pSUPER.neo vector (OligoEngine) and verified according to the manufacturer's procedure [16]. Preparing and multiplying plasmid DNA containing the shRNA sequence took place in a culture of competitive Escherichia coli bacteria. After verification of correctness of the cloning using enzymatic hydrolysis and dideoxy sequencing, the designed shRNA silencing the STAT3 gene was used for transfection of human eukaryotic cell line T24 (transitional urinary bladder cancer cell line ATCC: HTB4).

\section{Cell cultures and silencing expression of STAT3 gene}

The human cancer cell line T24 culture was cultivated in standard conditions (temperature $37^{\circ} \mathrm{C}$, humidity $95 \%$, atmosphere containing 95\% air and 5\% $\left.\mathrm{CO}_{2}\right)$. The culture was cultivated in the Hera-Cell incubator (Heraeus). Cells were grown in RPMI-1640 medium containing L-glutamine (PAA Laboratories $\mathrm{GmbH}$ ), with the addition of $10 \%$ FBS (fetal bovine serum) (PAA Laboratories $\mathrm{GmbH}$ ). The doubling time of the cells in the culture was approximately 22 hours. The condition of the cell culture was measured using microscopic analysis (Axiovert inverted microscope, Zeiss). The cells' lifespan was measured in the Neubauer hemocytometric chamber using $0.1 \%$ trypan blue in physiological saline (Sigma).

Transfection of the cells was carried out with lipofection technique, using Lipofectamine ${ }^{\circledR}$ factor. The experiment was conducted according to the producer's guidelines (Invitrogen).

\section{Evaluation of RNA interference} at the transcript level

The STAT3 mRNA expression level in the native and transfected cells was evaluated using the real-time RT-PCR method based on commercially available probes and primers for amplification reactions (Applied Biosystems). For calculations, the Ct technique was used. As the reference gene, $\beta$-actin was applied. The control culture consisted of non-transfected cells from the T-24 cell line and the cells transfected with interfering, nonsense sequence SCR (scrambled), which was not complementary to any of the human genes.

\section{Evaluation of proliferation and apoptosis activation in cancer cells}

In order to check how silencing the expression of the STAT3 gene changes the susceptibility of transfected cells to apoptosis, a qualitative method (DAPI nucleic acid staining) and quantitative evaluation of the degradation level of genomic DNA were carried out. For this purpose, an immunoenzymatic technique of detection of internucleosomal products of apoptotic DNA decay, Cell Death Detection ELISA Plus Assay (Roche Applied Science), was used. As the apoptosis inducer, the topoisomerase-etoposide inhibitor in the concentration of $20 \mu \mathrm{M}$ was applied [17]. To examine changes in the cell proliferation rate the MTT assay was performed.

\section{Results}

The siRNA sequence silencing STAT3 expression was designed using the SIRNA program from the EMBOSS software package [14]. As an initial file we used mRNA for the STAT3 gene from the GenBank database [15] with reference number NM_003150 (4953 nucleotides in length).

The program suggested 274 silencing sequences with the score in the range 10-1. After analyzing all suggested sequences above 9 scores and above $50 \%$ of GC (guaninecytosine) pairs, the sequence 5'aagattgacctagagacccactc 3' was chosen.

The chosen sequence has shown complementarity to all three known transcriptional variants of the STAT3 gene of the human genome:

- to the mRNA sequence of the third transcriptional variant of the human STAT3 gene (reference number NM_213662.1):

\section{Query 1 AAGATTGACCTAGAGACCCACTC 23 \\ | | | | | | | | | | | | | | | | | | | | | | \\ Sbjct 1483 AAGATTGACCTAGAGACCCACTC 1505}

- to the MRNA sequence of the second transcriptional variant of the human STAT3 gene (reference number NM_003150.3):

\section{Query 1 AAGATTGACCTAGAGACCCACTC 23 \\ | | | | | | | | | | | | | | | | | | | | 1591 \\ - to the mRNA sequence of the first transcriptional variant of the human STAT3 gene (reference number NM_139276.2):}

\section{Query 1 AAGATTGACCTAGAGACCCACTC 23 Sbjct 1591 AAGATTGACCTAGAGACCCACTC 1613}

In the description above, the phrase Query means the designed sequence of interfering RNA and the phrase Sbjct means the fragment complementary to all transcriptional variants (1-3) of MRNA for the STAT3 gene. Based on the chosen sequence, proper oligonucleotide constructions were 


\section{GATCCCCGATTGACCTAGAGACCCACTTCAAGAGAGTGGGTCTCTAGGTCAATCTTTTTA | | || || || ||||||||||||||||||||||||||||||||||||||||||||||| GGGCTAACTGGATCTCTGGGTGAAGTTCTCTCACCCAGAGATCCAGTTAGAAAAATTCGA}

Fig. 1. Designed and created sequence used for creating the pSUPER vector, which actively expresses shRNA molecules complementary to STAT3 mRNA. The sequences recognized by appropriate restriction enzymes and used for cloning designed shRNA to the pSUPER vector are marked in red. Target siRNA sequence is marked in blue, nonsense sequence is marked in green, and the loop sequence of final shRNA is marked in violet

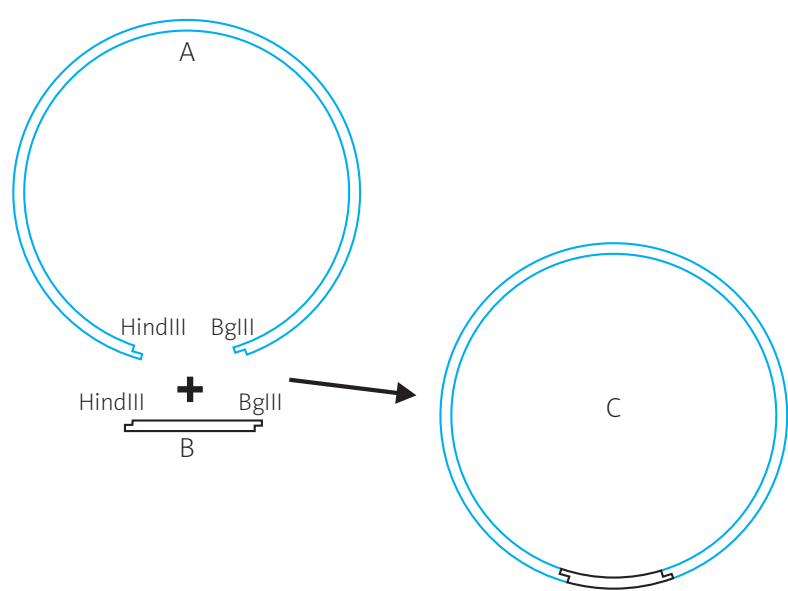

Fig. 2. The process of cloning the insert to the linearized plasmid pSUPER.neo. A - linearized plasmid with sticky ends after digestion with HindIII and BgllI enzymes, B - insert obtained by hybridization of oligonucleotide forward and reverse shRNA having sticky ends complementary to the ends of the plasmid after linearization; C - ligation product used for shRNA expression

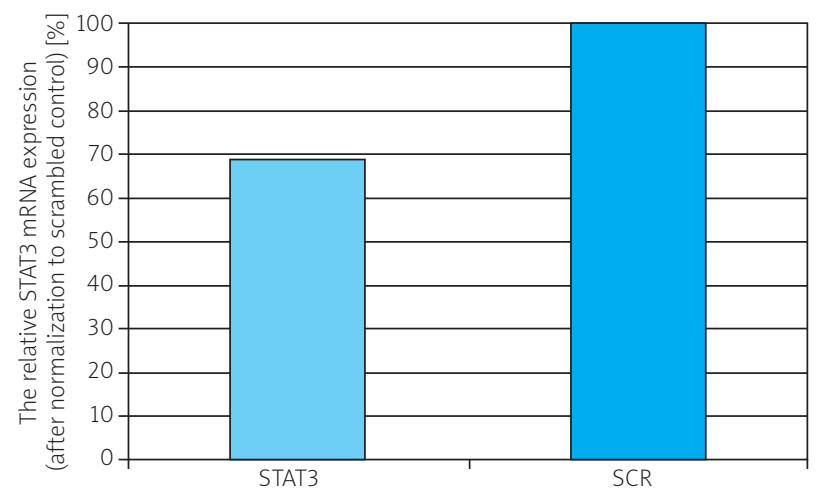

Fig. 3. Changes in the expression level of mRNA for STAT3 gene in the T24 cell culture 24 hours after inserting the sequence silencing STAT3 gene with reference to control culture (SCR)

designed. These constructions included both sense strain and antisense strain of the target gene. Additionally, for cloning purposes, the nucleotide systems were added. As a result, the final construction included restriction sites and the element determining the creation of short hairpin RNA conformation (shRNA). As the cloning was conducting in the plasmid DNA vector, the design of a double strained structure including forward and reverse oligonucleotides was taken into consideration. The forward and reverse oligonucleotides corresponded to sense and antisense fragment sequences of the targeted gene.
All designed sequences including the silencing region of mRNA for STAT3 are described below:

- oligonucleotide STAT3shF (forward): 5'GATCCCCGATTGACCTAGAGACCCACTTCAAGAGAGTGGG TCTCTAGGTCAATCTTTTTA 3'

content of GC pairs: 46.7\%; molecular weight: $18366.00 \mathrm{~g} / \mathrm{mol}$; TM: $72.8^{\circ} \mathrm{C}$

- oligonucleotide STAT3shR (reverse):

5’AGCTTAAAAAGATTGACCTAGAGACCCACTCTCTTGAAG

TGGGTCTCTAGGTCAATCGGG 3'

content of GC pairs: 46.7\%; molecular weight: $18464.00 \mathrm{~g} / \mathrm{mol}$; $\mathrm{T}_{\mathrm{M}}: 72.8^{\circ} \mathrm{C}$.

As a result of hybridization of 60-nucleotide forward and reverse sequences, a double strained oligonucleotide was created (Fig. 1). This oligonucleotide had so-called "sticky 5' ends" with a sequence complementary to the cloning site in the pSUPER.neo vector (Fig. 2) and to the STAT3 transcript. The STAT3 transcript binds to the region from 1569 to 1591 nucleotides.

In order to evaluate the gene expression at the mRNA level, the method of indirect characterization of gene expression was used. This method compares the number of mRNA copies of the examined gene to the number of mRNA copies of the $\beta$-actin gene, which is used as an endogenic control. 24 hours after cell transfection with the shRNA construction for STAT3 and with the control construction (shRNA-SCR), the level of mRNA expression for the STAT3 gene was assessed using real-time RT-PCR. Using the $\Delta \Delta C$ method of calculations we established that the mRNA expression level for the STAT3 gene in the T24 cell line (with silenced STAT3) was on average at the level of $68.3 \%$ of the mRNA expression level of STAT3 in the T24 cell line transfected with the shRNA-SCR construction (Fig. 3).

With reference to phenotypic changes which are the result of silencing of STAT3 transcript activity, the evaluation of proliferation and apoptosis induction changes in the T24 cells was carried out. The cells which were transfected with the PSUPER vector including the sequence silencing expression of mRNA for the STAT3 gene were compared to the cells which were not transfected. Figure 4 shows the T24 cells stimulated to apoptosis with etoposide and stained with DAPI fluorescent dye. In the group of non-transfected cells (A) apoptotic bodies were not observed. In the group of cells with silenced STAT3 gene stimulated to apoptosis with etoposide (D) the highest number of apoptotic bodies was observed in comparison to other experimental groups of cells $(B, C)$. The increase of apoptosis activation was also assessed quantitatively based on the increase of the amount of internucleosomal cleavage of genomic DNA of transfected cells, which were stimulated with topoisomerase-etoposide inhibitor. In analogy to the result obtained through fluorescent 

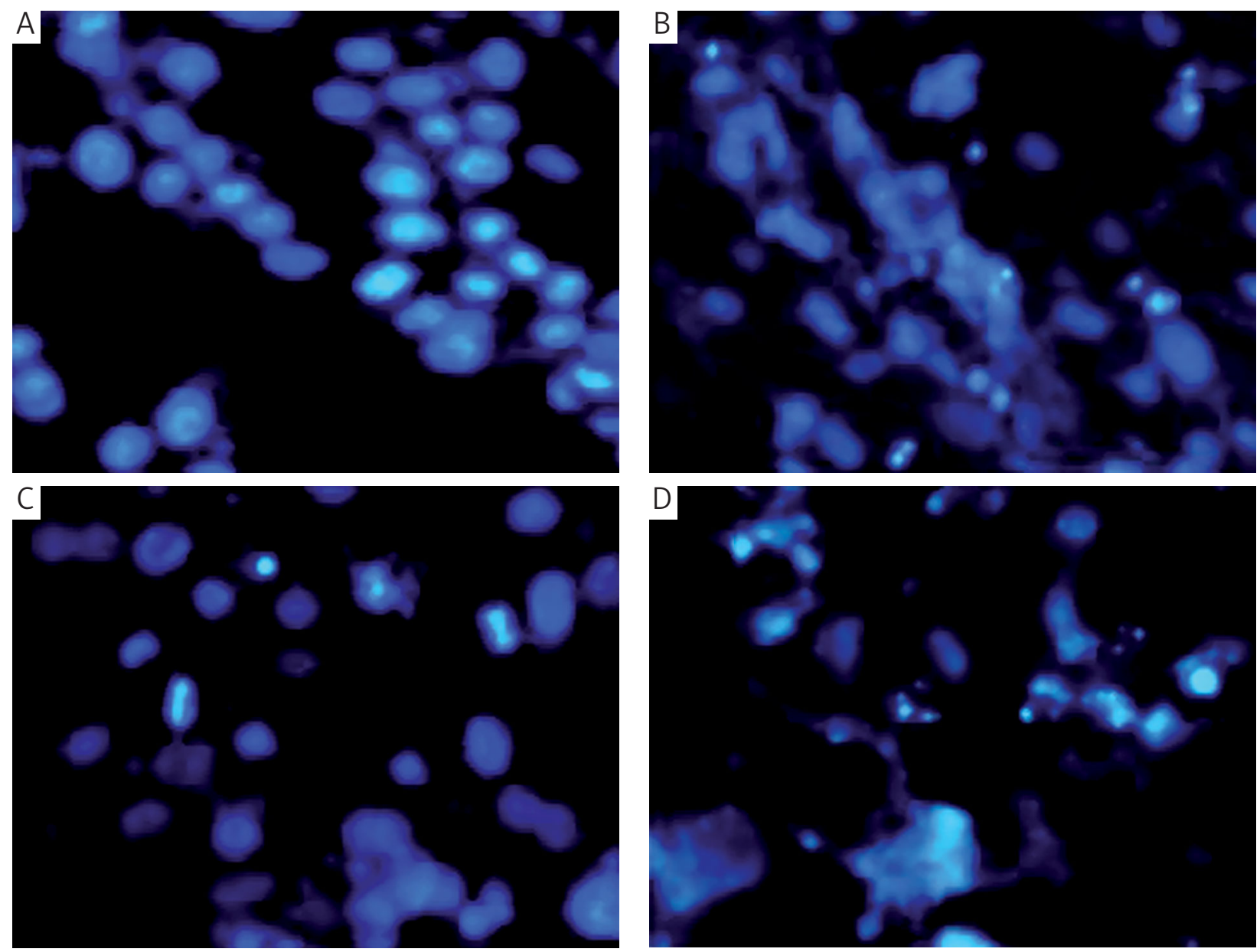

Fig. 4. Evaluation of apoptosis in T24 cell culture by DAPI staining. A - non-transfected cells; B - non-transfected cells treated with etoposide; C - cells transfected with shRNA/STAT3 construction; D - cells transfected with shRNA/STAT3 construction and treated with etoposide

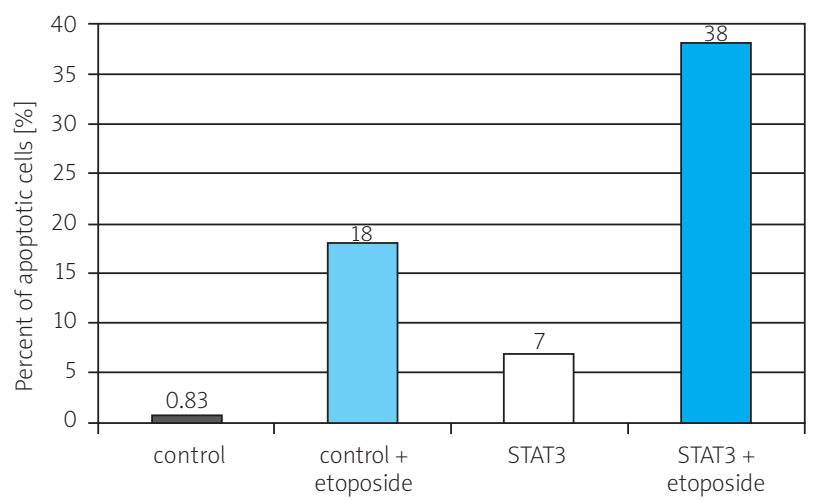

Fig. 5. The percentage of apoptotic cells in T24 cell culture estimated by measuring the level of DNA fragmentation using the Cell Death Detection ELISA Plus Assay

staining, the largest percentage of apoptotic cells was observed in the group of cells transfected with the STAT3/shRNA construction and treated with etoposide (Fig. 5). The differences in the absorbance obtained in the MTT assay for each examined group allowed us to quantify the changes in cell proliferation. As shown in Fig. 6, the proliferation rate of cells with silenced STAT3 in combination with etoposide was decreased approximately 2-fold compared to the untreated control. In both cases, statistically significant differences in cell apoptosis induction and proliferation

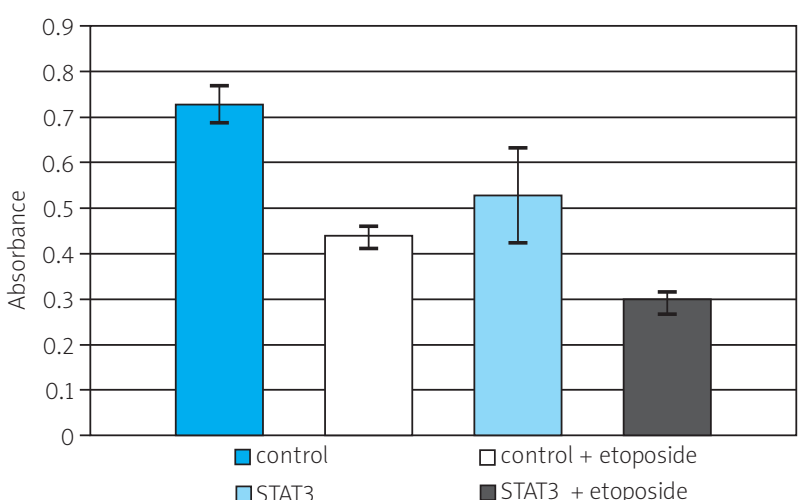

Fig. 6. Decrease in the absorbance signal connected with reduced proliferation rate in MTT assay conducted in T24 cell culture

were observed (respectively: $p=0.0376$ for apoptosis induction and $p=0.0318$ for changes of proliferation level).

\section{Discussion}

Activation of STAT3 contributes to activation of several genes such as matrix metalloproteinases MMP-2 and MMP-9, cyclin D1, VEGF and survivins, cytokines (including IL-6), chemokines and cyclooxygenase 2 (COX 2). Increased signaling through the STAT3 proteins and related abnormalities can promote the development of neoplasm [18, 19]. 
Until now, the strategies of blocking STAT3 protein function include the tyrosine kinase inhibitors [20-23], attempts of using antisense oligonucleotides which can block STAT3 protein synthesis [24, 25], and RNA interference [26].

Many publications show the constitutive activation of STAT3 proteins in etiopathogenesis of many malignancies, for example: hypernephroid carcinoma, papillary renal carcinoma, urothelial carcinoma, prostate cancer, lung cancer, ovarian cancer, pancreatic cancer, head and neck malignancies, leukemias and malignant melanoma [27-29].

Increased signaling with STAT proteins leads to proliferation stimulation and suppression of apoptosis. It is connected with constant stimulation of tyrosine kinases joined with the receptors for growth factors and cytokines (for example SRC, JANUS kinases, BCR-ABL, receptors with inner kinase domain) [30, 31].

Within earlier mentioned types of malignancies, urinary bladder cancer is one of the most frequent. What is more, it often recurs and it readily gives rise to metastases [32]. There is a high correlation between the overexpression of STAT3 protein and the process of carcinogenesis. As a result, there is a need to find a therapy in which we can silence that overexpression.

Within the last decade, the post transcriptional silencing of gene expression has been widely discussed in the context of applying it in the diagnostics and therapy of many illnesses, including malignancies. Post transcriptional silencing of gene expression can be developed by antisense oligonucleotides, ribozymes or small interfering RNA molecules [20-26]. RNA interference (RNAi) can be achieved by applying two different kinds of RNA molecules: synthetic small interfering RNA (siRNA) and short RNA with hairpin conformation (shRNA) synthesized in an expression vector brought into the cell. Despite the fact that both siRNA and shRNA are able to create specific gene silencing, they show different silencing effectiveness. What is more, after induction from the vector, shRNA molecules cause lower, non-specific effects within genes that are not targeted. This difference is probably caused by the different mechanisms of including siRNA and shRNA in the RNAi pathway. If using a vector with insert coding shRNA (under the control of polymerase II), the original transcript is polyadenylated. Apart from that, siRNAs need to be used in higher concentrations than ShRNA molecules if we desire effects at the same level. This makes siRNA molecules more susceptible to causing non-specific effects. What is more, unprotected siRNA can be degraded in the physiological processes [33].

To sum up, shRNA molecules have advantages which make them more useful and more effective than siRNAs, such as lower frequency of unspecific effects, the ability to silence a larger number of genes without a need to increase its concentration, durability of the effect and the ability to create the induced expression. For this reason, in the present work we silenced the expression of a gene coding the STAT3 protein using an expression vector which contained and expressed the insert including the desired STAT3/shRNA molecules.

24 hours after transfection of the T24 cell line with the expression vector a decline of the mRNA level was observed. It was about $68 \%$ of the initial level in the examined cancer cell line T24. The investigations conducted by Rivat and co- workers show a similar decline of the STAT3 expression to ours (approximately 28\%) [34]. However, their investigational model was related to the large bowel cancer cell line HCT8/S11 and their siRNA sequence was binding to the STAT3 mRNA fragment in the region from 1571 to 1589 nucleotides.

For silencing STAT3 expression, Lifang and co-workers used three SiRNA constructions, which bound to the following regions: from 1571 to 1580 nucleotides (first construction), from 438 to 456 nucleotides (second one) and from 2144 to 2162 nucleotides (third construction) of STAT3 mRNA. The authors assessed the STAT3 mRNA expression level 72 hours after transfection, using the Northern blot method. For the second construction, the mRNA expression decreased to the level of $25 \%$ and for the third construction to the level of $20 \%$, related to the control culture. However, for the first construction, no silencing effect on STAT3 mRNA expression was observed. Based on both the obtained results and analysis of secondary structure in bindings regions for STAT3 mRNA, the authors advanced the hypothesis that silencing STAT3 gene expression using siRNA constructions is dependent on localization of the siRNA sequence binding region in the target mRNA, and in consequence, possible secondary structures forming in this region.

In the present work, out of all suggested (by the siRNA program) interfering siRNA sequences, we chose the sequence targeting the mRNA fragment which includes the DNA binding domain (DBD). It is a key region for STAT3 protein proper function.

Although the changes in the mRNA level were not so noticeable, the desired effect of the RNAi strategy with reference to changes of phenotype of cancer cells was observed. Namely, a statistically significant increase in the amount of apoptotic cells after etoposide stimulation was observed in cells with the silenced STAT3 gene. That is the STAT3 transcriptional factor which activates genes coding antiapoptotic BCL-2 protein, vascular endothelial growth factor (VEGF) or antiapoptotic survivin protein. Finally, it blocks expression of the gene coding P-53 protein, which is a very important effector in the process of apoptosis [35].

As the induction and the course of the process of apoptosis bring fast changes in the cells, we can suppose that even a transient and short-lived silencing effect on STAT3 expression can bring the expected effect - increased elimination of cancer cells.

The sequence designed in the present work is effective, but the effect of silencing STAT3 expression does not last long and its efficiency is low. This may be caused by cancer cells' "defense", used when they are denied of STAT3 influence and when the expression of shRNA-STAT3 construction is switched off, for example in the epigenetic pathway. In order to increase the efficiency of silencing STAT3 expression in cancer cells and obtain the most profitable therapeutic results, it is worth verifying further shRNA-STAT3 constructions designed for other localizations within STAT3 mRNA.

The designed and synthesized genetic construction coding and expressing shRNA interfering molecules for the target transcript, mRNA for STAT3, shows the ability to suppress expression of the gene coding the STAT3 transcriptional factor. Along with decreased transcriptional activity of the STAT3 
gene, the induction of apoptosis and decreased proliferation of modulated urinary bladder cancer cell line T24 can be observed.

The research was funded by the statutory finance resources KNW-1-117/09 and KNW-1-151/10. This work was supported by the European Community from the European Social Fund within the RFSD 2 project.

\section{References}

1. Darnell JE Jr. STATs and gene regulation. Science 1997; 277: 1630-5. 2. Darnell JE Jr, Kerr IM, Stark GR. Jak-STAT pathways and transcriptional activation in response to IFNs and Rother extracellular signaling proteins. Science 1994; 264: 1415-21.

3. Bromberg J, Darnell JE Jr. The role of STATs in transcriptional control and their impact on cellular function. Oncogene 2000; 19: 2468-73.

4. Ramana CV, Chatterjee-Kishore M, Nguyen H, Stark GR. Complex roles of Stat1 in regulating gene expression. Oncogene 2000; 19: 2619-27.

5. Kotenko S V, Pestka S. Jak-Stat signal transduction pathway through the eyes of cytokine class II receptor complexes. Oncogene 2000; 19: 2557-65.

6. Lin JX, Leonard WJ. The role of Stat5a and Stat5b in signaling by IL-2 family cytokines. Oncogene 2000; 19: 2566-76.

7. Schindler C, Darnell JE Jr.Transcriptional responses to polypeptide ligands: the JAK-STAT pathway. Annu Rev Biochem 1995; 64: 621-51.

8. Hirano T, Ishihara K, Hibi M. Roles of STAT3 in mediating the cell growth, differentiation and survival signals relayed through the IL-6 family of cytokine receptors. Oncogene 2000; 19: 2548-56.

9. Smithgall TE, Briggs SD, Schreiner S, Lerner EC, Cheng H, Wilson MB. Control of myeloid differentiation and survival by Stats. Oncogene 2000; 19: 2612-8.

10. Mui AL. The role of STATs in proliferation, differentiation, and apoptosis. Cell Mol Life Sci 1999; 55: 1547-58.

11. Bromberg JF, Wrzeszczynska MH, Devgan G, Zhao Y, Pestell RG, Albanese C, Darnell JE Jr. Stat3 as an oncogene. Cell 1999; 98: 295-303.

12. Buchholz F. RNA - Interference in Mammalian Cells. Euro Biotech News 2005; 4: 39-42.

13. Sui G, Soohoo C, Affar el B, Gay F, Shi Y, Forrester WC, Shi Y. A DNA vector-based RNAi technology to suppress gene expression in mammalian cells. Proc Natl Acad Sci U S A 2002; 99: 5515-20.

14. http://emboss.bioinformatics.nl/.

15. http://blast.ncbi.nlm.nih.gov/bl2seq/wblast2.cgi.

16. www.oligoengine.com.

17. Bednarek I. Etoposide - induced apoptosis of HL-60 cells may be enhanced by pyrrolidine dithiocarbamate (PDTC) (abstract). Gliwice Scientific Meeting 2004; 39.

18. Yu H, Pardoll D, Jove R. STATs in cancer inflammation and immunity: a leading role for STAT3. Nat Rev Cancer 2009; 9: 798-809.

19. Mantovani A, Allavena P, Sica A, Balkwill F. Cancer-related inflammation. Nature 2008; 454: 436-44.

20. Haridas V, Nishimura G, Xu ZX, Connolly F, Hanausek M, Walaszek Z, Zoltaszek R, Gutterman JU. Avicin D: a protein reactive plant isoprenoid dephosphorylates Stat 3 by regulating both kinase and phosphatase activities. PLoS One 2009; 4(5): e5578.

21. Meydan N, Grunberger T, Dadi H, et al. Inhibition of acute lymphoblastic leukaemia by a Jak-2 inhibitor. Nature 1996; 379: 645-8,

22. Molavi O, Ma Z, Mahmud A, Alshamsan A, Samuel J, Lai R, Kwon GS, Lavasanifar A. Polymeric micelles for the solubilization and delivery of STAT3 inhibitor cucurbitacins in solid tumors. Int J Pharm 2008; 347: 118-27.

23. Blaskovich MA, Sun J, Cantor A, Turkson J, Jove R, Sebti SM. Discovery of JSI-124 (cucurbitacin I), a selective Janus kinase/signal transducer and activator of transcription 3 signaling pathway inhibitor with potent antitumor activity against human and murine cancer cells in mice. Cancer Res 2003; 63: 1270-9.

24. Grandis JR, Drenning SD, Chakraborty A, Zhou MY, Zeng Q, Pitt AS, Tweardy DJ. Requirement of Stat3 but not Stat1 activation for epidermal growth factor receptor-mediated cell growth in vitro. I Clin Invest 1998; 102: 1385-92.
25. Leong PL, Andrews GA, Johnson DE, et al. Targeted inhibition of Stat3 with a decoy oligonucleotide abrogates head and neck cancer cell growth. Proc Natl Acad Sci U S A 2003; 100: 4138-43.

26. Li GH, Wei H, Lv SQ, Ji H, Wang DL. Knockdown of STAT3 expression by RNAi suppresses growth and induces apoptosis and differentiation in glioblastoma stem cells. Int J Oncol 2010; 37: 103-10.

27. Guo C, Yang G, Khun K, Kong X, Levy D, Lee P, Melamed J. Activation of Stat3 in renal tumors. Am J Transl Res 2009; 1: 283-90.

28. Gao L, Zhang L, Hu J, et al. Down-regulation of signal transducer and activator of transcription 3 expression using vector-based small interfering RNAs suppresses growth of human prostate tumor in vivo. Clin Cancer Res 2005; 11: 6333-41.

29. Ikuta K, Takemura K, Kihara M, et al. Overexpression of constitutive signal transducer and activator of transcription 3 mRNA in cisplatinresistant human non-small cell lung cancer cells. Oncol Rep 2005; 13: 217-22.

30. Samanta AK, Lin H, Sun T, Kantarjian H, Arlinghaus RB. Janus kinase 2: a critical target in chronic myelogenous leukemia. Cancer Res 2006; 66: 6468-72

31. Abou-Ghazal M, Yang DS, Qiao W, et al. The incidence, correlation with tumor-infiltrating inflammation, and prognosis of phosphorylated STAT3 expression in human gliomas. Clin Cancer Res 2008; 1329-47.

32. Pasin E, Josephson DY, Mitra AP, Cote RJ, Stein JP. Superficial bladder cancer: an update on etiology, molecular development, classification, and natural history. Rev Urol 2008; 10: 31-43.

33. Rao DD, Senzer N, Cleary MA, Nemunaitis J. Comparative assessment of siRNA and shRNA off target effects: what is slowing clinical development. Cancer Gene Ther 2009; 16: 807-9.

34. Rivat C, Rodrigues S, Bruyneel E, et al. Implication of STAT3 signaling in human colonic cancer cells during intestinal trefoil factor 3 (TFF3) - and vascular endothelial growth factor-mediated cellular invasion and tumor growth. Cancer Res 2005; 65: 195-202.

35. Sahu RP, Srivastava SK. The role of STAT-3 in the induction of apoptosis in pancreatic cancer cells by benzyl isothiocyanate. J Natl Cancer Inst 2009; 101: 176-93.

\section{Address for correspondence}

\section{Anna Galilejczyk}

Department of Biotechnology and Genetic Engineering

Medical University of Silesia

Narcyzów 1

41-200 Sosnowiec

e-mail: annagolda86@gmail.com

Submitted: $\quad 19.10 .2011$

Accepted: $\quad 01.06 .2012$ 